

International

Diabetes

Federation

\title{
Association between hemoglobin glycation index and hepatic steatosis in non-diabetic individuals
}

\author{
Teresa Vanessa Fiorentino ${ }^{a, 1}$, Maria Adelaide Marini ${ }^{b, 1}$, Elena Succurro ${ }^{a}$, \\ Francesco Andreozzi ${ }^{a}$, Angela Sciacqua ${ }^{a}$, Marta Letizia Hribal ${ }^{a}$, Francesco Perticone ${ }^{a}$, \\ Giorgio Sesti ${ }^{a, *}$ \\ ${ }^{a}$ Department of Medical and Surgical Sciences, Viale Europa, University Magna-Groecia of Catanzaro, 88100 Catanzaro, Italy \\ ${ }^{\mathrm{b}}$ Department of Systems Medicine, University of Rome Tor Vergata, 00133 Rome, Italy
}

\section{A R T I C L E I N F O}

Article history:

Received 29 May 2017

Received in revised form

26 August 2017

Accepted 19 September 2017

Available online 6 October 2017

\section{Keywords:}

Nonalcoholic fatty liver disease

Hemoglobin glycation index

Hepatic steatosis

Non-enzymatic protein glycation

\begin{abstract}
A B S T R A C T
Aims: Hemoglobin glycation index (HGI), which is the difference between the observed value of $\mathrm{HbA1}$ and the predicted $\mathrm{HbA1c}$ based on plasma glucose levels, represents a measure of the degree of non-enzymatic glycation of hemoglobin and it has been found to be positively associated with diabetic complications. Herein we investigated whether HGI is associated with hepatic steatosis and related biomarkers in subjects without diabetes.

Methods: 1120 White individuals without diabetes were stratified in quartiles according to HGI levels. Hepatic steatosis was diagnosed by ultrasonography.

Results: As compared with subjects in the lowest quartile of HGI those in the intermediate and high HGI groups displayed an unfavorable cardio-metabolic risk profile having significantly higher values of body mass index (BMI), waist circumference, $\%$ fat mass, total cholesterol, triglycerides, inflammatory markers such as high sensitivity $\mathrm{C}$ reactive protein, erythrocytes sedimentation rate, complement C3, platelets and white blood cell count, hepatic insulin resistance assessed by the liver IR index and lower concentrations of highdensity lipoprotein. HGI was positively associated with the biomarker of liver damage alanine aminotransferase, and fatty liver index, an indicator of hepatic steatosis. In a logistic regression analysis adjusted for age, gender and BMI individuals in the highest quartile of HGI exhibited a 1.6-fold increased odd of having hepatic steatosis (95\% CI: 1.03-2.41; $\mathrm{p}=0.03$ ) as compared with subjects in the lowest quartile of HGI.

Conclusions: Higher levels of HGI may identify subjects without diabetes at increased risk of having hepatic steatosis.
\end{abstract}

(c) 2017 Elsevier B.V. All rights reserved.

\section{Introduction}

Non-alcoholic fatty liver disease (NAFLD) represents a spectrum of progressive liver disorders ranging from hepatocellular steatosis to steatohepatitis, which in turn may lead to cirrhosis, and, ultimately, hepatocellular carcinoma [1,2]. NAFLD is the most common cause of liver disease in Western countries affecting up to $46 \%$ of the

\footnotetext{
* Corresponding author at: Dipartimento Scienze Mediche e Chirurgiche, Università “Magna-Græcia” di Catanzaro, Viale Europa, 88100 Catanzaro, Italy.

E-mail address: sesti@unicz.it (G. Sesti).

1 These authors equally contributed as first authors to the work. https://doi.org/10.1016/j.diabres.2017.09.017 0168-8227/@ 2017 Elsevier B.V. All rights reserved.
} 
general population, and its prevalence continues to rise worldwide mainly as a consequence of the constantly increasing prevalence of obesity and diabetes $[3,4]$.

A growing body of evidence suggests that NAFLD is strongly associated not only with impaired glucose metabolism and obesity [5-7] but also with an increased risk to develop diabetes mellitus [8-10], and an independent association between NAFLD and glycated hemoglobin (HbA1c) levels has been reported in several studies [11-14]. HbA1c is the gold standard for evaluating glucose control in patients affected by diabetes mellitus and for monitoring the efficacy of therapies $[15,16]$. Moreover HbA1c is commonly used also as a diagnostic test for diabetes and conditions at increased risk of diabetes (the so-called prediabetes) in accordance with American Diabetes Association (ADA) recommendation [17].

Importantly, a poor concordance between $\mathrm{HbA1c}$ and other parameters of glucose control is often observed in clinical practice. Indeed, $\mathrm{HbA1c}$ and other measures of glycaemia reflect different aspects of glucose metabolism, the former is an indicator of the non-enzymatic protein glycation which is dependent of glucose concentration into erythrocytes whereas the latter expresses the physiology of glucose in the extracellular compartment. A mathematical method to measure the discordance between $\mathrm{HbA1c}$ and the predicted value of HbA1c based on plasma glucose levels has been developed and termed hemoglobin glycation index (HGI) [18-21].

HGI is the difference between the observed value of HbA1 and the predicted HbA1c, which is calculated by inserting plasma glucose concentration into a population regression equation expressing the linear association between HbA1c and circulating glucose levels. HGI represents a measure of the degree of non-enzymatic glycation of hemoglobin and it has been found to be positively associated with diabetic complications [21,22]. In patients with type 1 diabetes participating to the Diabetes Control and Complications Trial (DCCT) it has been observed that a higher HGI, calculated by using mean blood glucose levels, was associated with a greater risk of retinopathy and nephropathy [21]. Furthermore, it has been recently reported that a higher HGI value, calculated by using fasting plasma glucose in patients with type 2 diabetes participating to the Action to Control Cardiovascular Risk in Diabetes (ACCORD) trial, was associated with diabetic complications and with greater mortality in the intensive treatment group [22]. However, whether HGI is associated with organ damage also in subjects without diabetes has not been investigated to date. Remarkably, non-enzymatic protein glycation is known to be one of the crucial players in the pathogenesis of NAFLD $[2,23,24]$. Nevertheless, no studies have been carried out to assess the correlation between HGI and NAFLD in subjects without type 2 diabetes. To address this issue, we sought to evaluate whether HGI is associated with hepatic steatosis and related biomarkers in a large cohort of adult White subjects participating to the CATAnzaro MEtabolic RIsk factors (CATAMERI) study $[14,25-27]$.

\section{Materials and methods}

The design of the CATAMERI study has been previously reported [14,25-27]. The study cohorts include 2055 participants used to estimate the linear relationship between fasting plasma glucose and HbA1c in the study population to calculate the predicted $\mathrm{HbA1C}$ value, and 1120 individuals in whom hepatic steatosis was assessed by ultrasonography. All participants were consecutively recruited at the Department of Medical and Surgical Sciences of the University "Magna Graecia" of Catanzaro [14,25-27]. Exclusion criteria included: history of type 1 or type 2 diabetes mellitus, heart failure, end stage renal disease, haemoglobinopathies or anemia, history of malignant disease, gastrointestinal diseases associated with bleeding or malabsorption, autoimmune diseases, acute or chronic infections, positivity for antibodies to hepatitis C virus (HCV) or hepatitis B surface antigen (HBsAg), accumulation diseases such as amyloidosis and hemochromatosis, history of drug abuse, self-reporting alcohol consumption of $>20$ g/day, and history of treatments known to induce liver injury including tamoxifen, glucocorticoids, tetracycline, estrogens, methotrexate, and amiodarone. After a 12 -h fasting, all individuals underwent anthropometrical evaluation including measurements of BMI, waist circumference, body composition assessed by bioelectrical impedance and readings of clinic blood pressure. A $75 \mathrm{~g}$ oral glucose tolerance test (OGTT) was performed with sampling for plasma glucose and insulin assays. All participants underwent hepatic ultrasonography with longitudinal, sub costal, ascending, and oblique scans performed by the same trained operator, who was blind to their clinical characteristics, using a Toshiba Aplio 50 ultrasound apparatus equipped with a $3.5-\mathrm{MHz}$ linear transducer [14]. The ultrasound criteria used to diagnose fatty liver included liver and kidney echo discrepancy, the presence of an increased liver echogenicity or "bright liver, poor echo penetration into the deep portion of the liver, and vascular blurring either singly or in combination. The protocol was approved by the Hospital ethical committee (Comitato Etico Azienda Ospedaliera "Mater Domini") and written informed consent was obtained from all participants in accordance with principles of the Declaration of Helsinki.

\subsection{Analytical determinations}

HbA1c was measured with high performance liquid chromatography using a National Glycohemoglobin Standardization Program (NGSP) certified automated analyzer (Adams HA-8160 HbA1C analyzer, Menarini, Italy). Total and high lipoprotein density (HDL) cholesterol, triglycerides, and glucose levels were assayed by enzymatic methods (Roche, Basel, Switzerland). Alanine aminotransferase (ALT) and aspartate aminotransferase (AST) levels were measured using the $\alpha$ ketoglutarate reaction, and gamma-glutamyltransferase (GGT) levels with the L-gamma-glutamyl-3-carboxy-4-nitroa nilide rate method (Roche, Basel, Switzerland). Serum insulin concentrations were determined with a chemiluminescencebased assay (Immulite ${ }^{\circledR}$, Siemens, Italy). High sensitivity C reactive protein (hsCRP) levels were assessed by an 
automated instrument (CardioPhase ${ }^{\circledR}$ hsCRP, Milan, Italy). White blood cell (WBC) count was determined using an automated particle counter (Siemens Healthcare Diagnostics ADVIA ${ }^{\circledR}$ 120/2120 Hematology System, Italy). Erythrocytes sedimentation rate (ESR) was measured automatically by the stopped-flow technique in a capillary microphotometer (Alifax Test 1 System Polverara, Italy) and complement C3 levels were evaluated by an automated nephelometric technology using the BN ${ }^{\mathrm{TM}} \mathrm{II}$ System analyzer (Siemens Healthcare, Italy).

\subsection{Calculation of HGI}

A random sub-sample of 2055 participants to the CATAMERI study was used to estimate the linear relationship between fasting plasma glucose and HbA1c. Of 2055 participants 1120 individuals were subjected to hepatic steatosis evaluation by ultrasonography. A predicted value of $\mathrm{HbA1c}$ was calculated by inserting fasting plasma glucose concentration into the sub-sample linear regression equation $\left(\mathrm{HbA1c}=0.0158^{*}\right.$ fasting glucose levels $(\mathrm{mg} / \mathrm{dl})+4.0311)$. HGI was calculated by subtracting the predicted value of HbA1c from the observed HbA1c level for all participants, as previously described $[22,27]$. Study participants (1120 subjects) were stratified into quartiles according to their HGI value.

\subsection{Other calculations}

The fatty liver index was calculated using the formula: $\left(\mathrm{e}^{\wedge}\right.$ $0.953^{*}$ loge (triglycerides) $+0.139^{*} \mathrm{BMI}+0.718^{*}$ loge $(\mathrm{GGT})+$ $0.053^{*}$ waist circumference -15.745$) /\left(1+\mathrm{e}^{\wedge} 0.953^{*}\right.$ loge (triglycerides $)+0.139^{*} \mathrm{BMI}+0.718^{*}$ loge $(\mathrm{GGT})+0.053^{*}$ waist circumference -15.745$)^{*} 100$ [28].

The liver insulin resistance index (Liver IR index) was computed using the following formula: $-0.091+(\log$ insulin AUC $0-120 \mathrm{~min} \times 0.400)+(\log$ fat mass $\% \times 0.346)-(\log$ HDL Choles terol $\times 0.408)+(\log$ BMI $\times 0.435)[29]$.

The Matsuda index of insulin sensitivity was computed as follows: $10,000 /$ square root of [fasting glucose ( $\mathrm{mmol} / \mathrm{L}) \mathrm{x}$ fasting insulin $(\mathrm{mU} / \mathrm{L})] \mathrm{x}$ [mean glucose $\mathrm{x}$ mean insulin during OGTT] [30].

\subsection{Statistical analysis}

Variables with skewed distribution including triglycerides, fasting and $2 \mathrm{~h}$-post load insulin, AST, ALT, GGT, hsCRP, and ESR were natural log transformed for statistical analyses. Continuous data are expressed as means \pm SD. Categorical variables were compared by $\chi^{2}$ test. Anthropometric and metabolic differences between groups were assessed after adjusting for age, gender and BMI using a general linear model with post hoc Fisher's least significant difference correction for pairwise comparisons. Relationships between variables were determined by Pearson's correlation coefficient (r). Partial correlation coefficients adjusted for age, and gender were computed between variables. A multivariate logistic regression analysis was used to determine the association between the study groups and liver steatosis. A multivariable linear regression analysis was performed in order to evaluate the independent contributions of the actual HbA1c and HGI to
NAFLD. The variance inflection factor (VIF) was higher than 5 indicating that multicollinearity among variables was a problem in the multiple regression models. A P value $<0.05$ was considered statistically significant. All analyses were performed using SPSS software programme Version 17.0 for Windows.

\section{Results}

Study population comprised 1120 subjects, of whom 530 (47\%) were male. The mean age was $47 \pm 12$ years and mean BMI was $29 \pm 6 \mathrm{~kg} / \mathrm{m}^{2}$. Anthropometric features and biochemical findings of the study population stratified according to quartiles of HGI value are shown in Table 1 . The cumulative frequency distribution of actual fasting plasma glucose and HbA1c are shown in Fig. 1A and B. The distribution of fasting plasma glucose was similar among the four study groups. By contrast, the frequency distribution of $\mathrm{HbA1c}$ was markedly different in the four groups.

Significant differences between the four study groups were observed with respect to age. Indeed, subjects with higher levels of HGI were more likely to be older than individuals with lower HGI. After adjusting for age and gender significant differences between low (quartile 1), intermediate (quartile 2 and 3), and high (quartile 4) HGI groups were observed in terms of BMI, fat mass and waist circumference with low, intermediate, and high HGI groups exhibiting graded increases in these anthropometric parameters.

By design $\mathrm{HbA1c}$ was significantly and positively associated with HGI, however no differences in fasting and $2 \mathrm{~h}$ post load glucose levels, area under the curve of glucose levels during the $120 \mathrm{~min}$ time period of OGTT (AUC Gluc $_{0-120}$ ) were observed between the four study groups. After adjusting for age, gender and BMI, subjects in the intermediate and high HGI groups displayed progressively higher levels of total cholesterol, triglycerides and lower levels of HDL cholesterol and insulin sensitivity assessed by the Matsuda index in comparison to individuals in the lowest quartile of HGI.

Notably after adjusting for age, gender and BMI, subjects in the intermediate and high HGI groups displayed a graded increase of all the inflammatory markers measured i.e. hsCRP, ESR, complement C3, platelets and WBC count in comparison to the individuals in the lowest quartile of HGI.

We found a positive and statistically significant relationship between HGI and the biomarker of liver damage ALT, and fatty liver index, an indicator of hepatic steatosis, with individuals in the intermediate and high HGI groups exhibiting progressively higher levels of ALT and fatty liver index in comparison to low HGI group. Individuals in the intermediate and high HGI groups displayed a graded increase in GGT and AST levels in comparison to the lowest HGI quartile, however it did not reach the statistical significance. Furthermore, after adjusting for age and gender we observed that liver IR index, an index of hepatic insulin resistance, strongly correlated with hepatic glucose production and hepatic steatosis, was progressively increased in the intermediate and high HGI 


\begin{tabular}{|c|c|c|c|c|c|c|}
\hline Variables & $\begin{array}{l}\text { Whole } \\
\text { studysubjects }\end{array}$ & $\begin{array}{l}1 \text { Quartile } \\
(-1.49 ;-0.221)\end{array}$ & $\begin{array}{l}2 \text { Quartile } \\
(-0.218 ;-0.0323)\end{array}$ & $\begin{array}{l}3 \text { Quartile } \\
(-0.0321 ; 0.178)\end{array}$ & $\begin{array}{l}4 \text { Quartile } \\
(0.183 ; 0.978)\end{array}$ & $P$ \\
\hline Gender (Male/Female) & $530 / 590$ & $137 / 146$ & $126 / 150$ & $130 / 154$ & $137 / 140$ & 0.74 \\
\hline Age (yrs) & $47 \pm 12$ & $44.6 \pm 13.2$ & $45.2 \pm 12.8$ & $46.5 \pm 12.3 \S$ &  & $<0.0001 \#$ \\
\hline BMI $\left(\mathrm{kg} / \mathrm{m}^{2}\right)$ & $29 \pm 6$ & $27.9 \pm 5.3$ & $28.8 \pm 5.5^{\S}$ & $29.3 \pm 5.9 \S \S$ & $30.7 \pm 6.2 \S \S \S$ & $<0.0001^{*}$ \\
\hline Waist circumference $(\mathrm{cm})$ & $100 \pm 14$ & $97 \pm 12$ & $98 \pm 14$ & $99 \pm 14 \S$ & $105 \pm 15 \S \S \S$ & $<0.0001^{*}$ \\
\hline Fat Mass (\%) & $32.1 \pm 10$ & $31.1 \pm 11$ & $31.5 \pm 10$ & $32.1 \pm 9$ & $34.2 \pm 8 \S \S \S$ & $0.03^{*}$ \\
\hline SBP (mm Hg) & $126 \pm 16$ & $126 \pm 17$ & $124 \pm 16$ & $125 \pm 18$ & $129 \pm 16$ & 0.45 \\
\hline DBP (mmHg) & $79 \pm 11$ & $79 \pm 11$ & $77 \pm 11$ & $78 \pm 11$ & $80 \pm 10$ & 0.25 \\
\hline Fasting glucose (mg/dl) & $92 \pm 11$ & $93 \pm 10$ & $92 \pm 11$ & $92 \pm 10$ & $92 \pm 12$ & 0.16 \\
\hline 2-h post-load glucose (mg/dl) & $121 \pm 31$ & $117 \pm 32$ & $118 \pm 31$ & $121 \pm 31$ & $130 \pm 30$ & 0.12 \\
\hline AUC 0-120 Glucose (mg/dl'h) & $273 \pm 60$ & $265 \pm 61$ & $264 \pm 57$ & $270 \pm 58$ & $291 \pm 59$ & 0.08 \\
\hline Fasting insulin $(\mu \mathrm{U} / \mathrm{ml})$ & $14 \pm 9$ & $12 \pm 7$ & $14 \pm 10$ & $13 \pm 8$ & $15 \pm 10$ & 0.75 \\
\hline 2-h insulin $(\mu \mathrm{U} / \mathrm{ml})$ & $102 \pm 90$ & $83 \pm 79$ & $102 \pm 80$ & $100 \pm 89$ & $120 \pm 90^{\S}$ & 0.08 \\
\hline $\begin{array}{l}\text { Matsuda insulin sensitivity index } \\
\left(\mathrm{mg} \times \mathrm{L}^{2} \times \mathrm{mmol}^{-1} \times \mathrm{mU}^{-1} \times \mathrm{min}^{-1}\right)\end{array}$ & $70 \pm 44$ & $78 \pm 48$ & $75 \pm 50$ & $67 \pm 48^{\S}$ & $59 \pm 39 \S \S \S$ & 0.02 \\
\hline HbA1c (\%) & $5.4 \pm 0.3$ & $5.1 \pm 0.3$ & $5.4 \pm 0.2 \S \S \S$ & $5.6 \pm 0.2 \S \S \S$ & $5.8 \pm 0.2 \S \S \S$ & $<0.0001$ \\
\hline Total cholesterol (mg/dl) & $199 \pm 38$ & $192 \pm 37$ & $200 \pm 37 \S$ & $200 \pm 36^{\S}$ & $204 \pm 39 \S \S$ & 0.03 \\
\hline $\mathrm{HDL}(\mathrm{mg} / \mathrm{dl})$ & $52 \pm 14$ & $53 \pm 14$ & $52 \pm 13$ & $52 \pm 13$ & $49 \pm 15^{\S}$ & 0.03 \\
\hline Triglycerides (mg/dl) & $120 \pm 69$ & $111 \pm 66$ & $115 \pm 68$ & $118 \pm 62$ & $137 \pm 76 \S \S \S$ & 0.005 \\
\hline hsCRP (mg/l) & $3 \pm 3$ & $2.5 \pm 3$ & $2.9 \pm 3^{\S}$ & $3.0 \pm 3 \S \S$ & 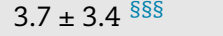 & 0.01 \\
\hline Complement C3 (g/l) & $1.17 \pm 0.2$ & $1.12 \pm 0.2$ & $1.15 \pm 0.2$ & $1.18 \pm 0.2$ & $1.22 \pm 0.2$ & 0.02 \\
\hline ESR $(\mathrm{mm} / \mathrm{h})$ & $11 \pm 9$ & $8.4 \pm 8$ & $10 \pm 8^{\S \S}$ & $11 \pm 9 \S \S \S$ & $14 \pm 10 \S \S \S$ & $<0.0001$ \\
\hline WBC count $\left(\times 10^{9} / 1\right)$ & $6774 \pm 1871$ & $6164 \pm 1509$ & $6793 \pm 1736$ §§§ & $6932 \pm 2152$ §§§ & $7214 \pm 1870 \S \S \S$ & $<0.0001$ \\
\hline Platelets count $\left(\times 10^{3} / 1\right)$ & $250 \pm 63$ & $230 \pm 59$ & $255 \pm 62$ §§§ & $252 \pm 64$ §§§ & $261 \pm 64 \S \S \S$ & $<0.0001$ \\
\hline ALT (UI/l) & $24 \pm 16$ & $23 \pm 13$ & $24 \pm 14$ & $24 \pm 15$ & $27 \pm 16^{\S \S}$ & 0.04 \\
\hline AST (UI/l) & $22 \pm 9$ & $20 \pm 7$ & $21 \pm 8$ & $22 \pm 11$ & $23 \pm 11$ & 0.13 \\
\hline GGT (UI/l) & $26 \pm 25$ & $24 \pm 20$ & $24 \pm 16$ & $26 \pm 32$ & $29 \pm 27$ & 0.35 \\
\hline Fatty liver index & $53.4 \pm 30$ & $46.3 \pm 30$ & $51.4 \pm 30^{\S}$ & $53.3 \pm 30 \S \S$ & $62.4 \pm 29$ §§§ & $<0.0001^{*}$ \\
\hline Liver IR index & $2.99 \pm 0.4$ & $2.85 \pm 0.4$ & $2.96 \pm 0.4^{\S}$ & $3.03 \pm 0.3 \S \S$ & $3.12 \pm 0.4 \S \S \S$ & $<0.0001^{*}$ \\
\hline Hepatic steatosis No (\%) & $283(28.4 \%)$ & $52(20.7 \%)$ & $64(27.6)$ & $73(28.2 \%)^{\S}$ & $94(37 \%)$ §§§ & 0.001 \\
\hline \multicolumn{7}{|c|}{$\begin{array}{l}\text { Data are means } \pm \text { SD. Fasting and 2-h insulin, triglycerides, AST, ALT, GGT, hsCRP, and ESR were log transformed for statistical analysis, but values in the table represent back transformation to the } \\
\text { original scale. Categorical variables were compared by } \chi^{2} \text { test. Comparisons between the four groups were performed using a general linear model with post hoc Fisher's least significant difference } \\
\text { correction for pairwise comparisons. P values refer to the trend (ANCOVA) with adjustment for age, gender, and BMI. \#P values refer to results after analyses with adjustment for gender. } P \text { values refer } \\
\text { to results after analyses with adjustment for gender and age. } \\
\text { BMI: body mass index; SBP: systolic blood pressure; DBP: diastolic blood pressure; hsCRP: high sensitivity C reactive protein; ALT: alanine aminotransferase; AST: aspartate aminotransferase; GGT: } \\
\text { gamma-glutamyltransferase; HDL: high density lipoprotein; ESR: erythrocytes sedimentation rate; WBC: white blood cell; Liver IR index: liver insulin resistance index. } \\
\$ P<0.05 \text { vs Quartile } 1 \text { of HGI. } \\
\$ \S P<0.01 \text { vs Quartile } 1 \text { of HGI. } \\
\$ \$ \S P<0.001 \text { vs Quartile } 1 \text { of HGI. }\end{array}$} \\
\hline
\end{tabular}



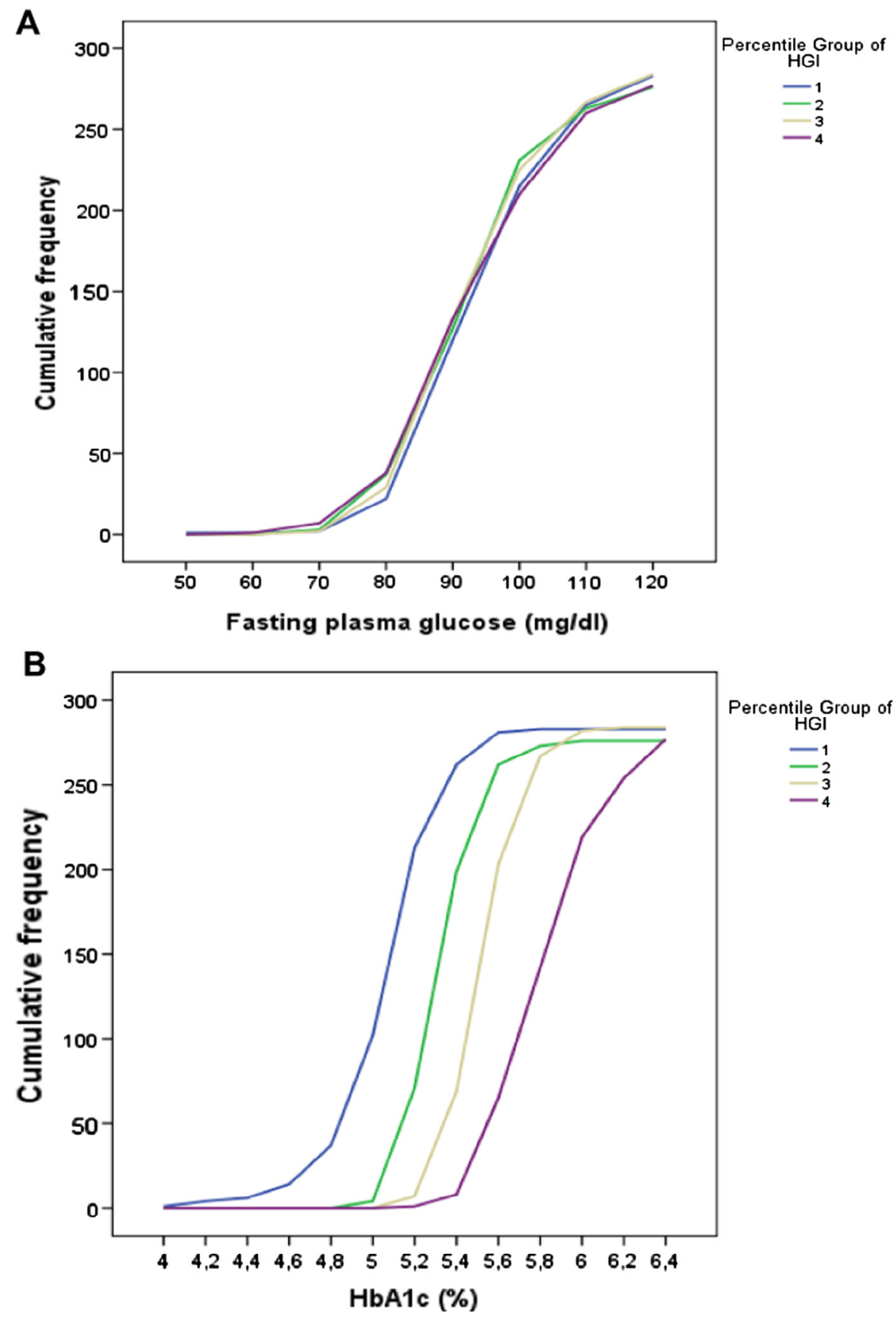

Fig. 1 - Cumulative frequency distribution of fasting plasma glucose levels in quartile groups of HGI (A). Cumulative frequency distribution of the observed HbA1c levels in HGI groups (B).

groups in comparison to the lowest HGI quartile. Accordingly, we observed a progressive increase in the prevalence of hepatic steatosis diagnosed by ultrasonography in low, intermediate, and high HGI groups (Table 1).

Age and gender adjusted univariate correlations between HGI levels and anthropometric and metabolic variables in the whole study population are shown in Table 2. HGI was positively correlated with BMI, waist circumference, total cholesterol, triglycerides, $2 \mathrm{~h}$ post-load glucose, HbA1c, fasting and $2 \mathrm{~h}$ post-load insulin levels and inversely associated with HDL concentrations and insulin sensitivity degree assessed by Matsuda index. Moreover a positive and significant relationship was found between $\mathrm{HGI}$ and the inflammatory markers hsCRP, ESR, complement C3, platelets and WBC count. Importantly HGI was positively associated with ALT, AST and GGT concentrations, and with fatty liver index and liver IR index levels.

A logistic regression analysis adjusted for age, and gender was used to determine the association between the four HGI groups and liver steatosis. As compared with individuals in the lowest quartile of HGI, those in the highest quartile of HGI had a significantly 2.2-fold increased odd of having hepatic steatosis (Table 3). Neither age nor gender were associated with increased risk of hepatic steatosis. After adding 
Table 2 - Age and gender adjusted univariate correlations between HGI and anthropometric and metabolic variables.

\begin{tabular}{|c|c|c|}
\hline & HGI & $P$ \\
\hline & $r$ & $P$ \\
\hline BMI $\left(\mathrm{kg} / \mathrm{m}^{2}\right)$ & 0.18 & $<0.0001$ \\
\hline Waist circumference $(\mathrm{cm})$ & 0.18 & $<0.0001$ \\
\hline Total cholesterol (mg/dl) & 0.07 & 0.02 \\
\hline HDL cholesterol (mg/dl) & -0.10 & 0.001 \\
\hline Triglycerides (mg/dl) & 0.13 & $<0.0001$ \\
\hline Fasting glucose (mg/dl) & -0.06 & 0.06 \\
\hline 2-h glucose (mg/dl) & 0.11 & 0.001 \\
\hline $\mathrm{HbA1c}(\%)$ & 0.89 & $<0.0001$ \\
\hline Fasting insulin $(\mu \mathrm{U} / \mathrm{ml})$ & 0.12 & $<0.0001$ \\
\hline 2-h insulin $(\mu \mathrm{U} / \mathrm{ml})$ & 0.12 & $<0.0001$ \\
\hline Matsuda Insulin Sensitivity index $\left(\mathrm{mg} \times \mathrm{L}^{2} \times \mathrm{mmol}^{-1} \times \mathrm{mU}^{-1} \times \min ^{-1}\right)$ & -0.15 & $<0.0001$ \\
\hline hsCRP (mg/l) & 0.19 & $<0.0001$ \\
\hline Complement C3 (g/l) & 0.18 & $<0.0001$ \\
\hline ESR $(\mathrm{mm} / \mathrm{h})$ & 0.24 & $<0.0001$ \\
\hline WBC count $\left(\times 10^{9} / 1\right)$ & 0.24 & $<0.0001$ \\
\hline Platelets count $\left(\times 10^{3} / 1\right)$ & 0.18 & $<0.0001$ \\
\hline ALT (UI/l) & 0.11 & $<0.0001$ \\
\hline AST (UI/l) & 0.09 & 0.003 \\
\hline GGT (UI/l) & 0.08 & 0.01 \\
\hline Fatty liver index & 0.22 & $<0.0001$ \\
\hline Liver IR index & 0.26 & $<0.0001$ \\
\hline
\end{tabular}

Table 3 - Odds ratios ( $95 \%$ CI) by multiple logistic regression models for hepatic steatosis in relation to HGI groups.

\begin{tabular}{|c|c|c|c|}
\hline \multirow{2}{*}{ Study groups } & \multirow[b]{2}{*}{ OR } & \multicolumn{2}{|c|}{ Hepatic steatosis } \\
\hline & & $95 \% \mathrm{CI}$ & $\mathrm{P}$ \\
\hline \multicolumn{4}{|l|}{ Model 1} \\
\hline Quartile 1 of HGI(reference category) & 1 & - & - \\
\hline Quartile 2 of HGI & 1.46 & $0.96-2.23$ & 0.076 \\
\hline Quartile 3 of HGI & 1.49 & $0.99-2.25$ & 0.053 \\
\hline Quartile 4 of HGI & 2.18 & $1.45-3.27$ & $<0.0001$ \\
\hline \multicolumn{4}{|l|}{ Model 2} \\
\hline Quartile 1 of HGI(reference category) & 1 & - & - \\
\hline Quartile 2 of HGI & 1.29 & $0.84-1.97$ & 0.247 \\
\hline Quartile 3 of HGI & 1.31 & $0.84-2.02$ & 0.231 \\
\hline Quartile 4 of HGI & 1.57 & $1.03-2.41$ & 0.03 \\
\hline \multicolumn{4}{|l|}{ Model 3} \\
\hline Quartile 1 of HGI(reference category) & 1 & - & - \\
\hline Quartile 2 of HGI & 1.36 & $0.88-2.11$ & 0.162 \\
\hline Quartile 3 of HGI & 1.38 & $0.91-2.11$ & 0.13 \\
\hline Quartile 4 of HGI & 1.58 & $1.04-2.43$ & 0.03 \\
\hline
\end{tabular}

BMI to the logistic regression model in addition to age and gender, the odd of subjects in the highest quartile of HGI to have hepatic steatosis was attenuated (1.6-fold), but it remained significant (Table 3). Similar results were obtained when waist circumference was included in the logistic regression model in addition to age and gender (Table 3).

\section{Discussion}

Individuals with $\mathrm{HbA1c}$ values persistently lower or higher than expected relatively to their blood glucose levels have been identified in several studies where blood glucose was estimated based on fasting plasma glucose [22], self- 
monitored blood glucose [18,19,31,32], continuous glucose monitoring data [20,33], and classified as high or low glycators. HGI is a measure of the disagreement between the observed value of HbA1c and the one predicted on the basis of blood glucose levels and it is thought to be an indicator of the degree of hemoglobin glycation. Hempe et al. [18] and McCarter et al. [21] have observed that a higher HGI level, calculated using self-glucose monitoring data to predict the value of $\mathrm{HbA1c}$, is associated with a greater risk of developing nephropathy and retinopathy in patients with type 1 diabetes. Several studies have demonstrated a positive association between micro- and macrovascular complications of type 2 diabetes and glycation gap, an analogue measure of the discordance between the observed and the predicted levels of HbA1c, calculated as HGI except that fructosamine concentrations replace blood glucose levels in the formula used to calculate predicted HbA1c [34-37]. An increased HGI level, calculated using fasting plasma glucose concentration to predict $\mathrm{HbA1c}$ value, has been recently shown to identify a subgroup of the ACCORD population with a greater risk of diabetic complications, hypoglycemia and total mortality [22]. Moreover we have recently found that higher HGI levels may identify subjects with an increased risk of vascular atherosclerosis also among individuals without diabetes [27].

In the present cross-sectional study, for the first time, we report an association between HGI and liver steatosis in subjects not affected by diabetes. We observed that individuals with higher HGI levels have an increased risk to have hepatic steatosis assessed by ultrasonography as compared to individuals with lower HGI independently of confounders such as BMI, age and gender. In addition subjects with higher HGI levels displayed a worse metabolic risk profile having greater levels of BMI, visceral adiposity, triglycerides and total cholesterol, liver biomarkers, such as ALT, hsCRP, fatty liver index and lower levels of HDL cholesterol and insulin sensitivity assessed by Matsuda index in comparison to subjects with lower HGI. Furthermore, we found a positive association between HGI and inflammatory status, indeed subjects with higher HGI levels exhibited increased levels of inflammatory biomarkers including hsCRP, C3 complement, ESR, WBC and platelets count in comparison to individuals in the low HGI group. Importantly, no significant differences in fasting and post-load glucose concentrations were observed between the study groups, suggesting that the association between HGI and NAFLD is independent of other measures of glucose homeostasis.

The pathophysiological mechanisms involved in the associations between HGI and NAFLD are still indefinite. HGI has been shown to reflect the burden of AGEs in the tissues [38], raising the possibility that subjects with high HGI have increased levels of AGEs in the liver. There is accumulating evidence that activation of RAGE downstream pathway by AGEs may promote pro-inflammatory reactions and impair insulin signaling, thus contributing to the development and progression of NAFLD $[23,24]$. Hepatic insulin resistance, is known to play a crucial role in hepatic fat accumulation $[2,8,39]$. On the other hand ectopic lipid deposition in the liver has been shown to activate pro-inflammatory pathways and promote insulin resistance, thus leading to a vicious circle that promotes NAFLD development. Accordingly we observed a greater degree of hepatic insulin resistance, estimated by liver IR index, a validated surrogate index of hepatic insulin resistance [29], in subjects with higher HGI as compared with those having a lower value of HGI.

Chronic inflammation is thought to be a core component of NAFLD [2,40-42]. Indeed, inflammation may impair insulin signalling, exacerbate liver fatty infiltration, induce endoplasmic reticulum stress and oxidative stress, promoting eventually progression of NAFLD to more advanced forms of liver disease [40-42]. We observed that subjects having a higher value of HGI exhibited increased levels of inflammatory biomarkers independently of other confounding factors suggesting that a greater degree of non-enzymatic glycation of intracellular proteins may play a pathogenic role in inducing low-grade chronic inflammation.

Moreover, visceral obesity and the consequent adipose metabolic dysfunction represent important risk factors for the development and progression of NAFLD [43,44]. Obesity may lead to an imbalanced production of pro- and antiinflammatory adipokines secreted from adipose tissue, which contributes to the pathogenesis of NAFLD. In particular a decreased release of adiponectin, an adipokine known to exert anti-inflammatory and insulin-sensitizing effects, has been found to play a crucial role in the pathogenesis of NAFLD $[43,44]$. Accordingly we found that HGI levels were positively associated with measures of visceral obesity such as BMI, waist circumference and \% fat mass.

Taken together our results support the idea that HGI may be a useful parameter to identify among non-diabetic individuals with similar levels of HbA1c those with a "high glycation" phenotype and an increased risk of having hepatic steatosis and related metabolic abnormalities, who may mostly benefit from lifestyle or pharmacological interventions aimed to prevent or delay adverse clinical outcomes.

The present study has some limitations. First, the diagnosis of liver steatosis was performed by ultrasound scanning rather than by invasive methods such as liver biopsy or expensive non-invasive approaches such as proton magnetic resonance spectroscopy or computed tomographic scanning. However, ultrasonography is the most commonly used method to diagnose hepatic steatosis in clinical practice and epidemiological studies. Second, all biochemical variables, including plasma glucose during OGTT and HbA1c, were measured once, a common limitation to most large epidemiological studies, and small day-to-day changes would be expected. Third, hepatic insulin resistance was assessed by liver IR index rather than by clamp studies combined with tracer techniques which are invasive and expensive methods and consequently not feasible in large scale studies. However, liver IR index has been previously validated against measurement of hepatic glucose production using tracers [29]. Furthermore, the information on alcohol intake was assessed by self-reported questionnaire, thus the true daily alcohol consumption may have been underestimated. Next, the present findings are only based on White individuals, and whether the results can also be extended to other ethnic groups is unknown. Indeed, previous studies have shown that HbA1c levels are higher among Blacks, Hispanics, American Indians, and Asian Americans compared to Whites [45]. Moreover, the study included only subjects without diabetes 
carrying at least one risk factor for type 2 diabetes, thus the results may not be generalizable to the general population or to patients with type 2 diabetes, and we cannot recommend the use of the CATAMERI regression equation in other populations. Additionally, the cross-sectional design of the study reflects only an association with prevalent and not incident NAFLD, and precludes us to draw any conclusion about cause and effect relationship between the degree of nonenzymatic glycation of intracellular proteins assessed by HGI and liver fat accumulation.

\section{Acknowledgments}

Author contributions: T.V.F. analyzed data and wrote the manuscript; M.A.M researched data, reviewed and edited the manuscript; E.S, F.A, A.S. researched data; M.L.H, F.P. contributed to the discussion and reviewed the manuscript; G.S. designed the study, analyzed the data and reviewed the manuscript.

\section{Conflict of Interest statement}

The authors declare that they have no conflict of interest.

Ethical approval: All procedures performed in this study were in accordance with the ethical standards of the institutional research committee and with the 1964 Helsinki declaration and its later amendments or comparable ethical standards.

Informed consent: Written informed consent was obtained from all individual participants included in the study.

\section{R E F E R E N C E S}

[1] Angulo P. Nonalcoholic fatty liver disease. N Engl J Med. 2002;346:1221-31.

[2] Dowman JK, Tomlinson JW, Newsome PN. Pathogenesis of non-alcoholic fatty liver disease. QJM 2010;103:71-83.

[3] Williams CD, Stengel J, Asike MI, Torres DM, Shaw J, Contreras $\mathrm{M}$, et al. Prevalence of nonalcoholic fatty liver disease and nonalcoholic steatohepatitis among a largely middle-aged population utilizing ultrasound and liver biopsy: a prospective study. Gastroenterology 2011;140:124-31.

[4] Bedogni G, Miglioli L, Masutti F, Tiribelli C, Marchesini G, Bellentani S. Prevalence of and risk factors for nonalcoholic fatty liver disease: the Dionysos nutrition and liver study. Hepatology 2005;42:44-52.

[5] Kantartzis K, Machann J, Schick F, Fritsche A, Häring HU, Stefan N. The impact of liver fat vs visceral fat in determining categories of prediabetes. Diabetologia 2010;53:882-9.

[6] Jimba S, Nakagami T, Takahashi M, Wakamatsu T, Hirota Y, Iwamoto Y. Prevalence of non-alcoholic fatty liver disease and its association with impaired glucose metabolism in Japanese adults. Diabet Med 2005;22:1141-5.

[7] Mohan V, Farooq S, Deepa M, Ravikumar R, Pitchumoni CS. Prevalence of non-alcoholic fatty liver disease in urban south Indians in relation to different grades of glucose intolerance and metabolic syndrome. Diabetes Res Clin Pract 2009;84:84-91.
[8] Yki-Järvinen H. Liver fat in the pathogenesis of insulin resistance and type 2 diabetes. Dig Dis 2010;28:203-9.

[9] Adams LA, Waters OR, Knuiman MW, Elliott RR, Olynyk JK. NAFLD as a risk factor for the development of diabetes and the metabolic syndrome: an eleven-year follow-up study. Am J Gastroenterol 2009;104:861-7.

[10] Shibata M, Kihara Y, Taguchi M, Tashiro M, Otsuki M. Nonalcoholic fatty liver disease is a risk factor for type 2 diabetes in middle-aged Japanese men. Diabetes Care 2007;30:2940-4.

[11] Ma H, Xu C, Xu L, Yu C, Miao M, Li Y. Independent association of $\mathrm{HbA1c}$ and nonalcoholic fatty liver disease in an elderly Chinese population. BMC Gastroenterol 2013;13:3.

[12] Calanna S, Scicali R, Di Pino A, Knop FK, Piro S, Rabuazzo AM, et al. Lipid and liver abnormalities in haemoglobin A1Cdefined prediabetes and type 2 diabetes. Nutr Metab Cardiovasc Dis 2014;24:670-6.

[13] Bae JC, Cho YK, Lee WY, Seo HI, Rhee EJ, Park SE, et al. Impact of nonalcoholic fatty liver disease on insulin resistance in relation to $\mathrm{HbA1c}$ levels in nondiabetic subjects. Am J Gastroenterol 2010;105:2389-95.

[14] Fiorentino TV, Andreozzi F, Mannino GC, Pedace E, Perticone M, Sciacqua A, et al. One-hour postload hyperglycemia confers higher risk of hepatic steatosis to HbA1c-defined prediabetic subjects. J Clin Endocrinol Metab 2016;101:4030-8.

[15] Gonen B, Robenstein AH, Rochman H, Tanega SP, Horwitz DL. Haemoglobin A I: an indicator of the metabolic control of diabetic patients. Lancet 1977;2:734-6.

[16] Koenig RJ, Peterson CM, Jones RL, Saudek C, Lehrman M, Cerami A. Correlation of glucose regulation and hemoglobin Ale in diabetes mellitus. N Engl J Med 1976;295:417-20.

[17] American Diabetes Association. Standards of medical care in diabetes-2016. Diabetes Care 2016;39:S13-23.

[18] Hempe JM, Gomez R, McCarter Jr RJ, Chalew SA. High and low hemoglobin glycation phenotypes in type 1 diabetes: a challenge for interpretation of glycemic control. J Diabetes Complications 2002;16:313-20.

[19] Soros AA, Chalew SA, McCarter RJ, Shepard R, Hempe JM. Hemoglobin glycation index: a robust measure of hemoglobin A1c bias in pediatric type 1 diabetes patients. Pediatr Diabetes 2010;11:455-61.

[20] Wilson DM, Xing D, Cheng J, Beck RW, Hirsch I, Kollman C, et al. Juvenile diabetes research foundation continuous glucose monitoring study group: persistence of individual variations in glycated hemoglobin: analysis of data from the Juvenile diabetes research foundation continuous glucose monitoring randomized trial. Diabetes Care 2011;34:1315-7.

[21] McCarter RJ, Hempe JM, Gomez R, Chalew SA. Biological variation in $\mathrm{HbA1c}$ predicts risk of retinopathy and nephropathy in type 1 diabetes. Diabetes Care 2004;27:1259-64.

[22] Hempe JM, Liu S, Myers L, McCarter RJ, Buse JB, Fonseca V. The hemoglobin glycation index identifies subpopulations with harms or benefits from intensive treatment in the ACCORD trial. Diabetes Care 2015;38:1067-74.

[23] Hyogo H, Yamagishi S. Advanced glycation end products (AGEs) and their involvement in liver disease. Curr Pharm Des 2008;14:969-72.

[24] Yamagishi S, Matsui T. Role of receptor for advanced glycation end products (RAGE) in liver disease. Eur J Med Res 2015;20:15.

[25] Fiorentino TV, Marini MA, Andreozzi F, Arturi F, Succurro E, Perticone $\mathrm{M}$, et al. One-hour postload hyperglycemia is a stronger predictor of type 2 diabetes than impaired fasting glucose. J Clin Endocrinol Metab 2015;100:3744-51.

[26] Fiorentino TV, Sesti F, Andreozzi F, Pedace E, Sciacqua A, Hribal ML, et al. One-hour post-load hyperglycemia combined with HbA1c identifies pre-diabetic individuals with 
a higher cardio-metabolic risk burden. Atherosclerosis 2016;253:61-9.

[27] Marini MA, Fiorentino TV, Succurro E, Pedace E, Andreozzi F, Sciacqua A, et al. Association between hemoglobin glycation index with insulin resistance and carotid atherosclerosis in non-diabetic individuals. PLoS ONE 2017;12:e0175547.

[28] Bedogni G, Bellentani S, Miglioli L, Masutti F, Passalacqua M, Castiglione A, et al. The Fatty Liver Index: a simple and accurate predictor of hepatic steatosis in the general population. BMC Gastroenterol 2006;6:33.

[29] Vangipurapu J, Stančáková A, Kuulasmaa T, Paananen J, Kuusisto JEGIR-RISC Study Group. A novel surrogate index for hepatic insulin resistance. Diabetologia 2011;54:540-3.

[30] Matsuda M, De Fronzo RA. Insulin sensitivity indices obtained from oral glucose tolerance testing: comparison with the euglycemic insulin clamp. Diabetes Care 1999;22:1462-70.

[31] Kamps JL, Hempe JM, Chalew SA. Racial disparity in A1C independent of mean blood glucose in children with type 1 diabetes. Diabetes Care 2010;33:1025-7.

[32] Hempe JM, Soros AA, Chalew SA. Estimated average glucose and self-monitored mean blood glucose are discordant estimates of glycemic control. Diabetes Care 2010;33:1449-51.

[33] Diabetes Research in Children Network (DirecNet) Study Group; Wilson, D.M., Kollman, C.: Relationship of A1C to glucose concentrations in children with type 1 diabetes: assessments by high-frequency glucose determinations by sensors. Diabetes Care. 31, 381-385 (2008).

[34] Rodríguez-Segade S, Rodríguez J, García Lopez JM, Casanueva FF, Camiña F. Estimation of the glycation gap in diabetic patients with stable glycemic control. Diabetes Care 2012;35:2447-50.

[35] Nayak AU, Nevill AM, Bassett P, Singh BM. Association of glycation gap with mortality and vascular complications in diabetes. Diabetes Care 2013;36:3247-53.

[36] Cohen RM, Holmes YR, Chenier TC, Joiner CH. Discordance between $\mathrm{HbA1c}$ and fructosamine: evidence for a glycosylation gap and its relation to diabetic nephropathy. Diabetes Care 2003;26:163-7.

[37] Rodríguez-Segade S, Rodríguez J, Cabezas-Agricola JM, Casanueva FF, Camiña F. Progression of nephropathy in type 2 diabetes: the glycation gap is a significant predictor after adjustment for glycohemoglobin (HbA1c). Clin Chem 2011;57:264-71.

[38] Felipe DL, Hempe JM, Liu S, Matter N, Maynard J, Linares C, et al. Skin intrinsic fluorescence is associated with hemoglobin $\mathrm{A}(1 \mathrm{c})$ and hemoglobin glycation index but not mean blood glucose in children with type 1 diabetes. Diabetes Care 2011;34:1816-20.

[39] Stefan N, Kantartzis K, Häring HU. Causes and metabolic consequences of fatty liver. Endocr Rev 2008;29:939-60.

[40] Zhao L, Chen Y, Tang R, Chen Y, Li Q Gong J, et al. Inflammatory stress exacerbates hepatic cholesterol accumulation via increasing cholesterol uptake and de novo synthesis. J Gastroenterol Hepatol 2011;26:875-83.

[41] Zhang XQ, Xu CF, Yu CH, Chen WX, Li YM. Role of endoplasmic reticulum stress in the pathogenesis of nonalcoholic fatty liver disease. World J Gastroenterol 2014;20:1768-76.

[42] Hribal ML, Procopio T, Petta S, Sciacqua A, Grimaudo S, Pipitone RM, et al. Insulin-like growth factor-1, inflammatory proteins, and fibrosis in subjects with nonalcoholic fatty liver disease. J Clin Endocrinol Metab 2013;98:E304-8.

[43] Rutkowski JM, Stern JH, Scherer PE. The cell biology of fat expansion. J Cell Biol 2015;208:501-12.

[44] Finelli C, Tarantino G. What is the role of adiponectin in obesity related non-alcoholic fatty liver disease? World J Gastroenterol 2013;19:802-12.

[45] Herman WH, Ma Y, Uwaifo G, Haffner S, Kahn SE, Horton ES, et al. Diabetes prevention program research group: differences in A1C by race and ethnicity among patients with impaired glucose tolerance in the Diabetes Prevention Program. Diabetes Care 2007;30:2453-7. 